\title{
Message Authentication Codes from Unpredictable Block Ciphers
}

\author{
Yevgeniy Dodis $^{1}$ and John Steinberger ${ }^{2}$ \\ ${ }^{1}$ Department of Computer Science, New York University \\ dodis@cs.nyu.edu \\ ${ }^{2}$ Department of Mathematics, University of British Columbia \\ jpsteinb@gmail.com
}

\begin{abstract}
We design an efficient mode of operation on block ciphers, SS-NMAC. Our mode has the following properties, when instantiated with a block cipher $f$ to yield a variable-length, keyed hash function $H$ :

(1) MAC Preservation. $H$ is a secure message authentication code (MAC) with birthday security, as long as $f$ is unpredictable.

(2) PRF Preservation. $H$ is a secure pseudorandom function (PRF) with birthday security, as long as $f$ is pseudorandom.

(3) Security against Side-Channels. As long as the block cipher $f$ does not leak side-channel information about its internals to the attacker, properties (1) and (2) hold even if the remaining implementation of $H$ is completely leaky. In particular, if the attacker can learn the transcript of all block cipher calls and other auxiliary information needed to implement our mode of operation.
\end{abstract}

Our mode is the first to satisfy the MAC preservation property (1) with birthday security, solving the main open problem of Dodis et al. [7] from Eurocrypt 2008. Combined with the PRF preservation (2), our mode provides a hedge against the case when the block cipher $f$ is more secure as a MAC than as a PRF: if it is false, as we hope, we get a secure variable-length PRF; however, even if true, we still "salvage" a secure MAC, which might be enough for a given application.

We also remark that no prior mode of operation offered birthday security against side channel attacks, even if the block cipher was assumed pseudorandom.

Although very efficient, our mode is three times slower than many of the prior modes, such as CBC, which do not enjoy properties (1) and (3). Thus, our work motivates further research to understand the gap between unpredictability and pseudorandomness of the existing block ciphers, such as AES.

\section{Introduction}

Most primitives in symmetric-key cryptography are built from block ciphers, such as AES. Typically, one models the block cipher as a fixed-input-length (FIL) pseudorandom permutation (PRP), and then builds a more complex variableinput-length (VIL) primitive under this assumption. For many such VIL primitives, like pseudorandom functions (PRFs), this strong assumption on the block

S. Halevi (Ed.): CRYPTO 2009, LNCS 5677, pp. 267-285, 2009.

(C) International Association for Cryptologic Research 2009 
cipher is justifiable. One exception here is the design of message authentication codes (MACs): since the resulting primitive only needs to be unpredictable, it would be highly desirable to only assume that the block cipher is unpredictable as well, as opposed to pseudorandom. Indeed, it seems that assuming the block cipher is unpredictable is a much weaker assumption than assuming full pseudorandomness: to break the latter, all one needs to do is to predict one bit of "random-looking" information about the block cipher with probability just a little over $1 / 2$, while the former requires one to fully compute the value of the block cipher on a new point with non-trivial probability. Thus, it is natural to ask the following central question of this work:

Question 1. Can one build an efficient variable-input-length MAC from a block cipher which is modeled as an unpredictable permutation (UP) on $n$-bits?

We will argue that no existing constructions are really satisfactory for achieving this natural goal. In order to discuss this precisely, we briefly recall some key quantities which determine the security of a construction. In this paper we consider only two types of adversaries: distinguishers, whose goal is to distinguish a function from an ideal primitive (as for PRFs and PRPs) and forgers, whose goal is to predict the value of the function on an un-queried message (as for MACs and UPs). As there often exist constant-query attacks using very long messages, the most important measure of an adversary's efficiency is the total length of messages that it queries. This upper bounds, among others, the number of queries made by the adversary. For functions that are built from a smaller primitive (such as, in all the cases we consider, a permutation), a more convenient efficiency measure is the number of queries one must make to the smaller primitive in order to evaluate the adversary's queries. In this section we let $q$ stand for the latter number, as opposed to the number of queries actually made by the adversary to its oracle.

Let $C$ be a function built from a block cipher $f$. The security 1$]=\varepsilon(q)$ of $C$ is the maximum advantage of an adversary for which the number of calls to $f$ necessary to compute the adversary's queries to $C$ does not exceed $q$. Thus, lower $\varepsilon$ implies better security. We write $\varepsilon_{\text {mac }}$ and $\varepsilon_{\text {prf }}$ to distinguish the security $C$ as MAC and PRF, respectively. Likewise the block cipher has a security $\varepsilon_{\text {up }}$ and $\varepsilon_{\text {prp }}$ as a UP and as PRP, respectively The rate of a VIL-function $C$ is the number of times the block cipher has to be called on each input block, so it measures the efficiency of $C$. We can now rephrase our goal as follows. Given a block cipher $f$ with UP security $\varepsilon_{\text {up }}$, construct a VIL-MAC $C$ such that:

(a) $C$ has small constant rate;

(b) the security $\varepsilon_{\text {mac }}$ of $C$ is as small as possible as a function of $\varepsilon_{\text {up }}$.

A good way to quantify the "goodness" of $\varepsilon_{\text {mac }}$ is to assess the maximum $q$ for which the achieved bound is meaningful, assuming that the block cipher has ideal

\footnotetext{
${ }^{1}$ Other parameters, such as the running time allowed to the adversary, may be relevant for the security, but these are not important here.

${ }^{2}$ In fact $\varepsilon_{\text {up }}=\varepsilon_{\text {mac }}$ for a block cipher, since these refer to the same notion, but we write $\varepsilon_{\text {up }}$ to emphasize the difference with $C$.
} 
security $\varepsilon_{\text {up }} \sim 1 / 2^{n}$ as an unpredictable permutation. For example, if $\varepsilon_{\text {mac }}=$ $O\left(\varepsilon_{\text {up }} \cdot q^{2}\right)$, then the bound is meaningful for $q$ up to $\sim 2^{n / 2}$, which matches the classical birthday bound typically achieved when one models the block cipher as a PRP. As argued by Preneel and van Oorschot [15], a simple extension attack shows that the birthday security is the best security one may hope to achieve by natural "iterative" constructions. On a positive, several elegant (iterative) constructions matching this bound are known, when modeling the block cipher as a PRP. On a negative, no existing constructions, iterative or otherwise, come even close to the birthday security when assuming UPs as opposed to PRPs. Thus, our "golden standard" to answer Question 1 will be to solve.

Question 2. Build an (iterative) VIL-MAC from UPs, having constant efficiency rate and (nearly) birthday security.

Jumping ahead, this will be our main result, therefore resolving the main open question of Dodis et al. 7 from Eurocrypt 2008. But first, let us survey what is known, to better understand the difficulties we will have to face, and also motivate our approach.

\subsection{Inapplicability of Existing Solutions}

There is a huge number of proposals for building a VIL-MAC out of a block cipher. Unfortunately, it turns out that most of them are insecure when instantiated with unpredictable block ciphers, - often despite having simple proofs of security when one models the block cipher as a PRP, - and the few that are secure, achieve extremely poor rate and/or security. In the full version [9, we give a detailed listing of many "failed" approaches to build an efficient VIL-MAC from an unpredictable block cipher. Here, we will only give a brief summary, concentrating on the approaches most relevant to our eventual solution.

In brief, the existing approaches in question include the following: (1) generic route from unpredictability to pseudorandomness [10, 13; (2) CBC-MAC [4, 14; (3) HMAC/NMAC [6, 3]; (4) various ad-hoc methods (e.g., iterating the truncated version of the block cipher); (5) hash-then-MAC using (almost) universal hashing [5, 3]; (6) hash-then-MAC using collision-resistant hashing; (7) Feistel Network [11,8; and (8) the current best method called "enhanced CBC" mode 7 . Of these, approaches (2), (3), (4) and (5) are completely insecure when instantiated with generic UPs (as opposed to PRPs!). This is simple to see for (3), (4) and (5), and was shown by An and Bellare 2 for the CBC-MAC (approach (2)). The generic approach (1) is secure, but very inefficient, which is not surprising.

Approach (6), using a collision-resistant hash function (CRHF) $H$ to hash the VIL message before applying a FIL-MAC, also works in principle, but is not satisfactory. In theory, the assumption that CRHFs exist is much stronger than the existence of UPs (or even PRPs); for example, there is a black-box separation [18, between these assumptions. Even in practice, where many hash functions are built from block ciphers, the resulting hash functions appear to require a larger security parameter than the "stand-alone" block ciphers they 
are built from. For example, while the industry standard AES has input length 128, no existing hash function with 128-bit output is considered secure (e.g., MD5 and related functions are broken [19]); in fact, NIST does not recommend using any hash function with output size below 256, including 160-bit SHA-1.

Weak Collision-Resistance. Thus, we would like to base security of the "hash-then-mac" approach on weaker hash functions than CRHFs. As was demonstrated by [6], the precisely correct notion for this task is that of Weak Collision Resistance (WCR). Such hash functions $H$ are keyed, and their key is part of the secret key for the resulting VIL-MAC. In terms of security, it should be infeasible for the attacker to come up with distinct inputs $x$ and $y$ such that $H(x)=H(y)$, even when given oracle access to $H$. An and Bellare [2] then showed that WCR hash functions have similar properties to CRHFs: in particular, the (strengthened) Merkle-Damgard transform gives a VIL-WCR hash function from a FIL-WCR hash function, which can then be used in "hash-thenmac" approach. Moreover, both security reductions are tight for our purposes.

Thus, to efficiently answer Question 1, it is sufficient to build a fixed-inputlength sufficiently compressing (say, two-to-one) WCR hash family. Indeed, this is the route of all existing solutions (e.g., approaches (7) and (8)), as well as our solution. However, to also answer Question 2 would additionally require a WCR hash with birthday security, which was not known prior to this work.

BUILDING WCR FROM UP. This question appears to be non-trivial. In particular, only two secure solutions were known prior to this work (approaches (7) and (8) above). First, Dodis and Puniya [8] showed how to construct a two-to-one FIL-WCR from $\omega(\log \kappa)$ independent UPs, where $\kappa$ is the security parameter. The construction applied $\omega(\log \kappa)$ rounds of the Feistel Network $\pi(x \| y)=(y \| f(y) \oplus x)$ to the $2 n$-bit input, each with a different UP $f$, and then truncated the last output in half. Moreover, they showed that $O(\log \kappa)$ rounds are generally insufficient for this task (extending the three-round counter-example of [2], and in sharp contrast to the setting of PRPs, where three rounds are already enough [11]). This means that the resulting super-constant rate $\omega(\log \kappa)$ of this particular construction cannot be improved, making it somewhat inefficient for practice. More significantly, the security of this construction proven by [8] was only $O\left(\varepsilon_{\text {up }} \cdot q^{6}\right)$, meaning that it can only be secure for at most $2^{n / 6}$ messages, which is unacceptable for $n=128$.

The best current WCR construction from UPs comes from the work of Dodis et al. 7], who made the surprisingly simple observation that the function $h(x \| y)=$ $f_{1}(x) \oplus f_{2}(y)$ is a two-to-one, rate-2 WCR hash function, assuming $f_{1}$ and $f_{2}$ are two independent UPs. This immediately gives a rate-2 VIL-MAC from UPs, which is very efficient, and is the first (and only) constant-rate solution to Question 1 known prior to this work. Unfortunately, the security of this WCR function (and the resulting VIL-MAC) is $O\left(\varepsilon_{\text {up }} \cdot q^{4}\right)$. Moreover, it is easy to see that this bound is actually tight. Thus, the construction can only be secure for at most $2^{n / 4}$ messages, again making it fall short of our goal of obtaining security up to $2^{n / 2}$. In fact, this question of achieving "birthday security" $2^{n / 2}$ (which is our Question 2) was the main open question posed in [7]. 


\subsection{Our Results}

In this work we resolve this question in the affirmative. Concretely, we construct a VIL-MAC, called SS-NMAC, from four independent UPs $f_{1}, \ldots, f_{4}$, which achieves rate 3 and security $\varepsilon_{\text {mac }} \approx O\left(\varepsilon_{\text {up }} q^{2} \log ^{2}(q)\right)$, meaning it can be secure for almost $2^{n / 2}$ messages. This is the first constant-rate MAC with birthday security built from an unpredictable block cipher. The construction of SS-NMAC is depicted in Figure 2, where the message is $x=x_{1} \ldots x_{\ell}$.

Our construction uses the WCR approach mentioned earlier: namely, it uses the (strengthened) Merkle-Damgard iteration of the $2 n$-bit to $n$-bit compression function $F(x \| y)=f_{1}(x) \oplus f_{3}\left(f_{1}(x) \oplus f_{2}(y)\right)$, which is shown in Figure 1 . This function was originally suggested by Shrimpton and Stam [17], who argued that $F$ is collision-resistant with birthday security, assuming that $f_{1}, f_{2}, f_{3}$ are public random functions (i.e., random oracles). In contrast, our main technical result (Theorem 1) shows that this function is (weakly) collision-resistant (with birthday security), even if $f_{1}, f_{2}, f_{3}$ are only (keyed) unpredictable functions.

We note that since any FIL-MAC is FIL-WCR (Lemma 4.4 [2]) it would suffice to prove the Shrimpton-Stam compression function is a good MAC in order to show it is WCR. However, as explained in the full version [9], the ShrimptonStam compression function is not a good enough MAC for our purposes, showing the necessity of directly proving WCR security.

COMPARISON WITH [17. On a technical level, both results appear similar. In both cases, assuming the adversary $A$ has oracle access to $f_{1}, f_{2}, f_{3}$, one has to argue that $A$ has low chance of finding a collision to $F$. However, the key difference is that the $f_{i}$ 's are assumed truly random in [17, whereas we can only assume unpredictable $f_{i}$ 's. In particular, while [17] could directly bound the probability of $A$ finding the collision in $F$ using an information-theoretic argument, we have to build an efficient reduction from the presumed collisionfinding attacker $A$ to a UP-forger $B$ forging one of the MACs. It is well known that such information-theoretic arguments often do not have direct analogs in the computational setting. To illustrate this more concretely, let us only discuss the most interesting such difficulty we had to resolve.

The key argument of [17] was a technical calculation, using factorials, binomials and various probability manipulations, that $A$ is unlikely to find an " $n$-way multi-collision" in the auxiliary function $h(x \| y)=f_{1}(x) \oplus f_{2}(y)$, when $f_{1}$ and $f_{2}$ are truly random functions. To adapt this (critical) part of the argument to our computational setting, we would have to take an efficient attacker $A^{\prime}$ capable of finding such a multi-collision in $h$ with probability $\varepsilon$, and turn it into a forger $B^{\prime}$ for either $f_{1}$ or $f_{2}$, succeeding with probability $\Omega\left(\varepsilon / q^{2}\right)$. As far as we could see, the probability calculations in [17] give no guidance of how to do such a reduction. And, indeed, finding such a reduction required a completely new approach, relating to a natural "balls-and-bins" game that we analyzed (see Lemma 1), and resulting in a very non-obvious construction of $B$. We discuss this construction in detail in Section 4.2, only mentioning that it gave us a better understanding of the security of the Shrimpton-Stam compression function, and even implicitly improved the probability calculations of [17] for the special 
case of truly random functions (corresponding to $\varepsilon=2^{-n}$ in our reduction). In particular, for the case of random functions we get a convenient closed form of $O\left(q^{2} \log ^{2}(q) / 2^{n}\right)$ for the collision resistance of the Shrimpton-Stam compression function, where, as per our convention for this section, $q$ is the total number of block cipher queries allowed (cf. Theorem 1).

Strong PRF PRESERVATION. We also notice that our new mode has the following desirable multi-property preservation guarantee advocated by [7]: if the block cipher is unpredictable, we get a MAC with message security roughly $2^{n / 2}$, while if it happens to be pseudorandom, we get a PRF with message security roughly $2^{n / 2}$. In other words, we expect and hope that practical block ciphers (such as AES) are in fact PRPs with good security. If our hope is correct, we would get a full-fledged pseudorandom function with good security; however, even if the block cipher turns out to be a much better MAC than it is a pseudorandom function, we still get a MAC with excellent security, which could be reassuring for many applications. Details are sketched in Section 5 .

More interestingly, even in the setting of PRPs, our SS-NMAC construction yields a more "leakage-resilient" VIL-PRF $H$ than the prior constructions. In particular, in Section 6 we show that the resulting PRF is secure even in the so called oracle cipher model, first considered by Dodis et al. 8. Recall, in the standard model for PRFs, the attacker only learns the output $H(x)$ of the PRF on input $x$, but does not learn any of the intermediate values, such as the inputs/outputs to the block cipher or any of the chaining variables. Indeed, this secrecy of the intermediate values is completely essential to the security of most standard constructions, such as CBC-MAC or the standard Luby-Rackoff transformation 11. In other words, these constructions are actually broken in the oracle cipher model, irrespective of the strength of the block cipher used (e.g., even with AES). In contrast, our SS-NMAC construction is a secure VIL-PRF - with (essentially) the same birthday security - even when the attacker learns all the intermediate values needed to obtain $H(x)$, except for what is done inside the actual block-cipher computations. More precisely, even if the attacker learns all the computation history of our SS-NMAC construction on a bunch of points (not including the internals of the block ciphers), the value of the function at any set of non-queried points looks random to the attacker. Thus, as long as the block cipher is implemented in a "leakage-resilient" way, the remaining implementation of SS-NMAC can be completely insecure with respect to side-channel attacks! We believe that the security in the oracle model is quite important, since we envision secure hardware-based implementation of block-ciphers, later composed with much less secure software-based solutions, to yield various more advanced VIL primitives. We also remark that none of the two previous PRF constructions in the oracle cipher model [8, 7] achieved anything close to birthday security.

Summary. To summarize, in addition to yielding a more secure VIL-MAC than prior constructions in the case when $\varepsilon_{\text {up }} \ll \varepsilon_{\text {prp }}$, our construction gives a more "leakage-resilient" (and equally secure!) VIL-PRF even when assuming $\varepsilon_{\text {prp }}$ is nearly as good as $\varepsilon_{\text {up }}$. Moreover, we only pay a small constant factor price in efficiency for these (significant) security enhancements. In Section 7 , we briefly 
discuss whether this slowdown is justifiable in practice, which ultimately calls for more research to understand the gap between unpredictability and pseudorandomness of the existing block ciphers, such as AES.

\section{Security Definitions}

We briefly recall the standard security notions for MACs and PRFs. In each case we are interested in resistance to chosen message attacks. For a MAC, an adversary succeeds if it can forge the MAC on an un-queried value. For a PRF, the adversary succeeds if it can distinguish the PRF from a truly random oracle. To measure an adversary's efficiency we count not only the number of oracle queries made but also the time and the total length of queried messages (as the oracles accept variable length inputs). In this section we use the variable $\tilde{q}$ to denote the number of queries made by the adversary to its oracle in order to emphasize the distinction from the variable $q$ used in Section 1, which was defined as the (distinct) number of block cipher calls necessary to evaluate those queries (the adversary does not have direct access to the block cipher). In later sections we maintain the spirit of this convention, using $\tilde{q}$ for queries made to VIL-functions and $q$ for queries made to FIL-functions (usually block ciphers).

A function family is a map $f:\{0,1\}^{\kappa} \times \operatorname{Dom}(f) \rightarrow\{0,1\}^{n}$ where $\operatorname{Dom}(f) \subseteq$ $\{0,1\}^{*}$. The strings in $\{0,1\}^{\kappa}$ are the keys of $f$ and we write $f_{k}(x)$ for $f(k, x)$ for $k \in\{0,1\}^{\kappa}$ and $x \in \operatorname{Dom}(f)$. The function $f_{k}$ is called a member of $f$.

For MACs we consider the following game, where $A$ is an adversary with oracle access to $f_{k}$ :

Game $\operatorname{Forge}(A, f)$ :

$k \leftarrow\{0,1\}^{\kappa} ;(x, y) \leftarrow A^{f_{k}}$

If $x \in \operatorname{Dom}(f), f_{k}(x)=y$ and $x$ was not a query of $A$ then $A$

wins, otherwise $A$ looses.

Following An and Bellare 2] we define the insecurity of $f$ as a MAC to be

$$
\operatorname{InSec}_{f}^{\operatorname{mac}}(t, \tilde{q}, \mu):=\max _{A} \operatorname{Pr}[A \text { wins } \operatorname{Forge}(A, f)]
$$

where the maximum is taken over all adversaries $A$ making at most $\tilde{q}$ queries whose total combined length is at most $\mu$ bits and of "running time" at most $t$. The "running time" is defined to be the total running time of the experiment, including the time necessary to compute the answers to $A$ 's queries. (The advantage of this definition is that a simulator running $A$ and computing the answer to $A$ 's queries from scratch has essentially the same running time $t$.)

For PRF security the game is modified by either giving $A$ access to a random $f_{k}$ or to a random oracle $g: \operatorname{Dom}(f) \rightarrow\{0,1\}^{n}$ with probability $\frac{1}{2}$ and $A$ wins if it correctly identifies whether its oracle is $f_{k}$ or $g$. Call this game 'Identifies $(A, f)$ '. Then

$$
\operatorname{InSec}_{f}^{\operatorname{prf}}(t, \tilde{q}, \mu):=\max _{A} \operatorname{Pr}[A \text { wins Identifies }(A, f)]-\frac{1}{2}
$$


where again the maximum is taken over all adversaries $A$ making at most $\tilde{q}$ queries of total length $\mu$ and of running time $t$, with the same convention concerning running time.

The proof finally uses the notion of "weak collision resistance" (WCR), which measures the collision resistance of a function only available as an oracle to the adversary. In the weak collision resistance game for the function family $f, A$ is given oracle access to a random $f_{k}$ and wins if it succeeds in querying $f_{k}$ on two distinct points $x, y$ such that $f_{k}(x)=f_{k}(y)$. Then $\operatorname{InSec}_{f}^{\mathrm{wcr}}(t, \tilde{q}, \mu)$ is defined similarly with respect to this game as $\operatorname{InSec}_{f}^{\operatorname{mac}}(t, \tilde{q}, \mu)$ is defined with respect to the game Forge $(A, f)$.

\section{The SS-NMAC Construction}

The basic SS-NMAC scheme is shown in Figure 2, The scheme uses the MerkleDamgard iteration of the $2 n$-bit to $n$-bit compression function of Shrimpton and Stam 17. shown in Figure 1. We start by describing this compression function.

The Shrimpton-Stam compression function. The Shrimpton-Stam compression function is a $2 n$-bit to $n$-bit compression function that uses calls to three different $n$-bit to $n$-bit primitives $f_{1}, f_{2}, f_{3}$. We write the compression function as $F\left[f_{1}, f_{2}, f_{3}\right]$ to emphasize its dependence on $f_{1}, f_{2}, f_{3}$. It is defined by

$$
F\left[f_{1}, f_{2}, f_{3}\right](x \| y)=f_{1}(x) \oplus f_{3}\left(f_{1}(x) \oplus f_{2}(y)\right)
$$

for any pair of $n$-bit strings $x, y$.

Shrimpton and Stam [17] proved that $F\left[f_{1}, f_{2}, f_{3}\right]$ has optimal (i.e. birthday) collision resistance if $f_{1}, f_{2}, f_{3}$ are random functions. They also conjectured that the construction remains collision resistant if $f_{i}(x)$ is replaced with $\pi_{i}(x) \oplus x$ where $\pi_{1}, \pi_{2}, \pi_{3}$ are random permutations, which would enable the construction to be implemented with fixed key block ciphers. This conjecture was verified by Rogaway and Steinberger [16.

For our purposes, the key property of $F\left[f_{1}, f_{2}, f_{3}\right]$ is that an adversary with oracle access to the $f_{i}$ 's can only learn $F\left[f_{1}, f_{2}, f_{3}\right](x \| y)$ on roughly as many inputs $x \| y$ as it makes queries. This should be contrasted for example to the compression function $h\left[f_{1}\right](x \| y)=f_{1}(x \oplus y)$ of the CBC MAC or the "xor

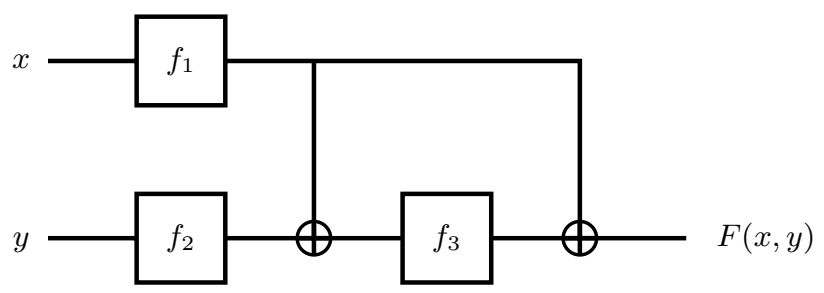

Fig. 1. The Shrimpton-Stam compression function. All wires carry $n$-bit values. 


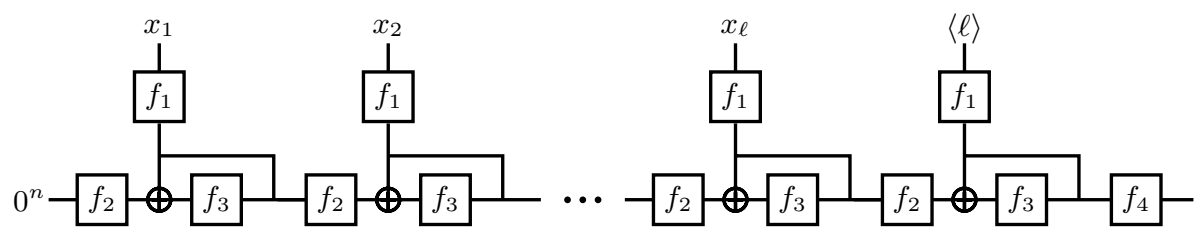

Fig. 2. The SS-NMAC mode of operation

compression function" $g\left[f_{1}, f_{2}\right](x \| y)=f_{1}(x) \oplus f_{2}(y)$ of the enciphered CBC construction of Dodis, Pietrzak and Puniya [7, where $f_{1}, f_{2}$ are again $n$-bit to $n$-bit functions. An adversary querying $h\left[f_{1}\right]$ can learn to evaluate $h\left[f_{1}\right]$ on $2^{n}$ inputs $x \| y$ in a single query; an adversary querying $g\left[f_{1}, f_{2}\right]$ can learn to evaluate $g\left[f_{1}, f_{2}\right]$ on $q^{2}$ inputs $x \| y$ in $q$ queries. Another compression function that could be used equally well in place of $F\left[f_{1}, f_{2}, f_{3}\right]$ is the LP231 compression function of Rogaway and Steinberger [16, which also uses three calls to $n$-bit to $n$-bit primitives. However we use $F\left[f_{1}, f_{2}, f_{3}\right]$ because it is simpler and sufficient for our purposes.

Iteration And PAdDing. First we define $\operatorname{PadAp}(x)$ to be $x 10^{k}\langle\ell\rangle$ where $k$ is the least integer such that $x 10^{k}$ has length a multiple of $n$, where $\ell$ is the number of $n$-bit blocks in $x 10^{k}$, and where $\langle\ell\rangle$ is $\ell$ written as an $n$-bit binary integer (messages with maximum length $2^{n}$ are sufficient for most applications). Appending $\langle\ell\rangle$ amounts to using Merkle-Damgard strengthening, which we do in order to keep our space of messages suffix-free. Any other suffix-free encoding of messages would do as well.

To iterate $F\left[f_{1}, f_{2}, f_{3}\right]$ we define the "SS-cascade" $G\left[f_{1}, f_{2}, f_{3}\right]$ of an $n \ell$-bit string $x=x_{1}\|\cdots\| x_{\ell}$ where each $x_{i}$ is an $n$-bit string by $G\left[f_{1}, f_{2}, f_{3}\right](x)=y_{\ell}$ where $y_{0}=0^{n}$ and $y_{k}=F\left[f_{1}, f_{2}, f_{3}\right]\left(x_{k} \| y_{k-1}\right)$ for $1 \leq k \leq \ell$. Finally, given an additional $n$-bit to $n$-bit function $f_{4}$ we define the SS-NMAC $H\left[f_{1}, f_{2}, f_{3}, f_{4}\right]$ by

$$
H\left[f_{1}, f_{2}, f_{3}, f_{4}\right](x)=f_{4}\left(G\left[f_{1}, f_{2}, f_{3}\right](x)\right) .
$$

for all $x \in \operatorname{Dom}(H):=\left\{\operatorname{PadAp}(y): y \in\{0,1\}^{*}\right\}$. See Figure 2, where $x=$ $x_{1}\|\cdots\| x_{\ell} \|\langle\ell\rangle$. Note that to query $H\left[f_{1}, f_{2}, f_{3}, f_{4}\right]$ on its domain an adversary must pad the input itself before giving it to the oracle. Thus queries must be at least $n$ bits long and the number of queries made by an adversary is upper bounded by $\mu / n$ where $\mu$ is the total length of messages queried by the adversary.

For the remainder of the paper we let $f:\{0,1\}^{\kappa} \times\{0,1\}^{n} \rightarrow\{0,1\}^{n}$ be an arbitrary, fixed function family. We consider $H$ as a function family of signature $\{0,1\}^{4 \kappa} \times \operatorname{Dom}(H) \rightarrow\{0,1\}^{n}$, where $H_{k_{1} k_{2} k_{3} k_{4}}(x):=H\left[f_{k_{1}}, f_{k_{2}}, f_{k_{3}}, f_{k_{4}}\right](x)$. Likewise we consider $F$ as a function family of signature $\{0,1\}^{3 \kappa} \times\{0,1\}^{2 n} \rightarrow$ $\{0,1\}^{n}$ defined by $F_{k_{1} k_{2} k_{3}}(x \| y)=F\left[f_{k_{1}}, f_{k_{2}}, f_{k_{3}}\right](x \| y)$. 


\section{Security of SS-NMAC as a MAC}

\subsection{Overview}

In this section we outline the proof that SS-NMAC is a secure MAC when $f_{1}, \ldots, f_{4}$ are secure MACs. The proof shows that $H$ is a secure MAC family if $f$ is a secure MAC family. In fact,

$$
\operatorname{InSec}_{H}^{\mathrm{mac}}(t, \tilde{q}, \mu) \leq\left(1+30 q^{2} \log ^{2}(q)\right) \cdot \mathbf{I n S e c}_{f}^{\mathrm{mac}}\left(t+O\left(q^{2} n\right), q, q n\right)
$$

where $q=\mu / n$ ( $\tilde{q}$ is inconsequent, though one automatically has $\tilde{q} \leq q)$. The $O\left(q^{2} n\right)$ difference in running time is due to the overhead of a simulator.

Like Dodis, Pietrzak and Puniya 7, our security proof follows the approach developed by An and Bellare 2, who reduce the VIL-MAC security to FIL-WCR security. In order to summarize their method in a convenient way we refer to the members of a function family as being MAC-secure or WCR-secure (see section 2 for the definition of WCR security) though security is really a property of the function family. An and Bellare reduce the MAC security of a VIL function to the WCR security of a FIL function in two steps:

Step 1: The composition of a secure FIL-MAC $f_{k}$ and a secure WCR function $G_{k^{\prime}}$ is a secure VIL-MAC $f_{k}\left(G_{k^{\prime}}(\cdot)\right)$ (Lemma $4.2[2]$ ). Applying this to the case where $f_{k}=f_{k_{4}}$ and $G_{k^{\prime}}=G\left[f_{k_{1}}, f_{k_{2}}, f_{k_{3}}\right]$ it therefore suffices to show that $G\left[f_{k_{1}}, f_{k_{2}}, f_{k_{3}}\right]$ is WCR-secure if $f$ is a secure MAC family in order to show that $H\left[f_{k_{1}}, f_{k_{2}}, f_{k_{3}}, f_{k_{4}}\right]=f_{k_{4}}\left(G\left[f_{k_{1}}, f_{k_{2}}, f_{k_{3}}\right]\right)$ is a secure MAC family.

Step 2: On a suffix-free domain of inputs the Merkle-Damgard iteration of a FIL-WCR compression function gives a VIL-WCR function (Lemma 4.3 [2]). Thus, by step 1, it suffices to show that the Shrimpton-Stam compression function $F\left[f_{k_{1}}, f_{k_{2}}, f_{k_{3}}\right]$ is FIL-WCR when $f$ is a secure MAC family.

Steps 1 and 2 give a qualitative description of An and Bellare's approach. Quantitatively, their Lemmas 4.2 and 4.3 imply that

$$
\operatorname{InSec}_{H}^{\mathrm{mac}}(t, \tilde{q}, \mu) \leq \operatorname{InSec}_{f}^{\mathrm{mac}}(t, q, q n)+\mathbf{I n S e c}_{F}^{\mathrm{wcr}}(t, q, 2 q n)
$$

where $q=\mu / n$. Since $\operatorname{InSec}_{f}^{\text {mac }}(t, q, q n) \leq \operatorname{InSec}_{f}^{\operatorname{mac}}\left(t+O\left(q^{2} n\right), q, q n\right)$ it therefore suffices to prove

$$
\operatorname{InSec}_{F}^{\mathrm{wcr}}(t, q, 2 q n) \leq 30 q^{2} \log ^{2}(q) \cdot \mathbf{I n S e c}_{f}^{\mathrm{mac}}\left(t+O\left(q^{2} n\right), q, q n\right)
$$

in order to prove (11). Inequality (3) is really the paper's main result, and we state it as a theorem:

Theorem 1. Let $f:\{0,1\}^{\kappa} \times\{0,1\}^{n} \rightarrow\{0,1\}^{n}$ and let $F:\{0,1\}^{3 \kappa} \times\{0,1\}^{2 n} \rightarrow$ $\{0,1\}^{n}$ given by $F_{k_{1} k_{2} k_{3}}(x \| y)=F\left[f_{k_{1}}, f_{k_{2}}, f_{k_{3}}\right](x \| y)=f_{k_{1}}(x) \oplus f_{k_{3}}\left(f_{k_{1}}(x) \oplus\right.$ $\left.f_{k_{2}}(y)\right)$. Then

$$
\operatorname{InSec}_{F}^{\mathrm{wcr}}(t, q, 2 q n) \leq 30 q^{2} \log ^{2}(q) \cdot \operatorname{InSec}_{f}^{\mathrm{mac}}\left(t+O\left(q^{2} n\right), q, q n\right) .
$$


The full proof of Theorem [1] is proven in the full version 9], but we give an outline in the next section.

Together with Lemmas 4.2 and 4.3 of [2, Theorem 1 implies inequality (3), which we restate as our theorem characterizing the MAC security of SS-NMAC:

Theorem 2. Let $f:\{0,1\}^{\kappa} \times\{0,1\}^{n} \rightarrow\{0,1\}^{n}$ and let $H:\{0,1\}^{4 \kappa} \times \operatorname{Dom}(H) \rightarrow$ $\{0,1\}^{n}$ be the SS-NMAC function family. Then, letting $q=\mu / n$,

$$
\operatorname{InSec}_{H}^{\operatorname{mac}}(t, \tilde{q}, \mu) \leq\left(1+30 q^{2} \log ^{2}(q)\right) \cdot \operatorname{InSec}_{f}^{\operatorname{mac}}\left(t+O\left(q^{2} n\right), q, q n\right) .
$$

\subsection{Proof Outline}

In this section we give a proof of Theorem 1 under several simplifying assumptions, which make our presentation considerably easier, while maintaining the key ideas of the full proof. Recall, we need to upper bound the WCR-insecurity of the Shrimpton-Stam compression function $F$ in terms of the MAC-insecurity of $f$. Equivalently, we must lower bound the MAC-insecurity of $f$ in terms of the WCR-insecurity of $F$. To do the latter, we show how an $\varepsilon$-collision-finding adversary $A$ for $F$ can be turned into a $\delta$-MAC-forging adversary $B$ for $f$, where $B$ uses the same number of queries but has chance of success $\delta=\Omega\left(\varepsilon / q^{2} \log ^{2}(q)\right)$.

First, instead of giving $A$ oracle access to $F\left[f_{1}, f_{2}, f_{3}\right]$, we directly give it oracle access to $f_{1}, f_{2}, f_{3}$, with $q$ queries allowed to each $f_{i}$. Clearly, such an adversary can simulate $q$ queries to $F$, so we only made $A$ more powerful. (Note, this strengthened attacker will be useful when we extend our argument to the "oracle cipher" model in Section [6]) Let us generally denote the inputs to $f_{1}, f_{2}, f_{3}$ by $x, y, z$, respectively, and also denote by $x_{1} \ldots x_{q}, y_{1} \ldots y_{q}$ and $z_{1} \ldots z_{q}$ the ordered inputs to $f_{1}, f_{2}, f_{3}$ supplied by $A$. As expected, the forger $B$ will simulate this adversary $A$ when trying to forge one of the $f_{i}$ 's, by using its own oracle to simulate the corresponding $f_{i}$, and simulating the other $f_{j}$ 's by picking their secret keys by itself and answering honestly.

Simplifying Assumptions. Before proceeding further, we state our simplifying assumptions on the behavior of $A$, which will make our construction of $B$ much simpler, while retaining the key ingredients of the general case.

- (No Collision in $f_{i}$ 's) For any distinct inputs $x_{r}$ and $x_{s}$ that $\mathcal{A}$ supplied to $f_{1}, f_{1}\left(x_{r}\right) \neq f_{1}\left(x_{s}\right)$. Similar conditions also hold for $f_{2}$ and $f_{3}$.

- (Query Order) All the calls to $f_{1}$ and $f_{2}$ are made by $A$ before any call to $f_{3}$ is made.

Let us briefly comment on these assumptions. The first assumption regarding the collisions in the $f_{i}$ 's is very minor, and is done for convenience only. Indeed, in the actual applications, the $f_{i}$ 's are permutations, so the assumption is trivially true. And even if the $f_{i}$ 's are arbitrary length-preserving MACs, the failure to satisfy our assumption with probability $\Omega(\varepsilon)$ trivially leads to a simple attacker $B$, forging the corresponding $f_{i}$ with probability $\Omega\left(\varepsilon / q^{2}\right)$, by simply guessing the indices $r, s \in\{1 \ldots q\}$ of the colliding queries. So the only "real" assumption we make is the Query Order Assumption. This assumption is provably impossible 
for the "initial" attacker who has oracle access to $F\left[f_{1}, f_{2}, f_{3}\right]$, as opposed to $f_{1}, f_{2}, f_{3}$ (since $f_{3}$ will be called on the first call there), and is even more unreasonable for the generalized attacker that can query the $f_{i}$ 's in any order it wants. However the assumption is used in a rather weak way in the proof sketch, as we will see, so that eliminating it only requires additional casework, and no significant new ideas.

Notation And Terminology. A ball is a pair $(x, y)$ where $x, y \in\{0,1\}^{n}$. A bin is a value $z \in\{0,1\}^{n}$. It is instructive to associate balls $(x, y)$ with the inputs to $F$, and the bins $z$ with the inputs to $f_{3}$. After $A$ makes $q$ queries $x_{1} \ldots x_{q}$ to $f_{1}$ and $y_{1} \ldots y_{q}$ to $f_{2}$, we get $Q=q^{2}$ potential balls $\left(x_{r}, y_{s}\right)$ "thrown" by $A$. In particular, we will say that such $\left(x_{r}, y_{s}\right)$ is placed into the bin $z=f_{1}\left(x_{r}\right) \oplus f_{2}\left(y_{s}\right)$, and let $\operatorname{Bin}(z)=\left\{\left(x_{r}, y_{s}\right): f_{1}\left(x_{r}\right) \oplus f_{2}\left(y_{s}\right)=z\right\}$ denote the set of balls placed into bin $z$. Notice, each query $x_{r}$ to $f_{1}$ allows the attacker to simultaneously place $j \leq q$ balls $\left(x_{r}, y_{1}\right), \ldots,\left(x_{r}, y_{j}\right)$, where $j$ is the number of queries to $f_{2}$ made so far. However, under our No Collision assumption of $f_{2}$, all these $j$ balls go to distinct bins $f_{1}\left(x_{r}\right) \oplus f_{2}\left(y_{s}\right)$, where $1 \leq s \leq j$. Similar discussion holds for the calls to $f_{2}$. Also, under our Query Order Assumption, the attacker $A$ places all $Q$ balls into the appropriate bins in the first stage, before making any of its queries $z_{1} \ldots z_{q}$ to $f_{3}$ in the second stage. And after each such query $z_{t}$ to $f_{3}, A$ learns the value of $F(x \| y)=f_{1}(x) \oplus f_{3}\left(z_{t}\right)$ precisely for all $(x, y) \in \operatorname{Bin}\left(z_{t}\right)$.

Back to Reduction. By our assumption, $A$ will find a collision $(x, y) \neq$ $\left(x^{\prime}, y^{\prime}\right)$ to $F$ with probability $\varepsilon$. Without loss of generality, we assume that $A$ makes the queries necessary to verify this collision. Thus, $x, x^{\prime} \in\left\{x_{1}, \ldots, x_{q}\right\}$, $y, y^{\prime} \in\left\{y_{1}, \ldots, y_{q}\right\}$, and $z, z^{\prime} \in\left\{z_{1}, \ldots, z_{q}\right\}$, where $z=f_{1}(x) \oplus f_{2}(y)$ and $z^{\prime}=$ $f_{1}\left(x^{\prime}\right) \oplus f_{2}\left(y^{\prime}\right)$. Notice, under our No Collision assumption on $f_{1}$ and $f_{2}$, we claim that $z \neq z^{\prime}$. Otherwise, $f_{3}(z)=f_{3}\left(z^{\prime}\right)$ and $f_{1}(x) \oplus f_{3}(z)=F(x \| y)=$ $F\left(x^{\prime} \| y^{\prime}\right)=f_{1}\left(x^{\prime}\right) \oplus f_{3}\left(z^{\prime}\right)$ imply that $f_{1}(x)=f_{1}\left(x^{\prime}\right)$, meaning that $x=x^{\prime}$. Then $f_{2}(y)=f_{1}(x) \oplus z=f_{1}\left(x^{\prime}\right) \oplus z^{\prime}=f_{2}\left(y^{\prime}\right)$, so $y=y^{\prime}$, meaning that $(x, y)=\left(x^{\prime}, y^{\prime}\right)$. Hence, the "colliding" bins $z$ and $z^{\prime}$ queried by $A$ must be distinct.

We now define a key parameter which will determine the behavior of our forger $B$ : the maximum bin size $m=\max _{z}|\operatorname{Bin}(z)|$ after the calls to $f_{1}$ and $f_{2}$ (the "filling" stage). We consider two complementary cases: (1) $A$ finds a collision and $m \leq \log (q)$, meaning that every bin $z$ contains at most $\log (q)$ balls after the calls to $f_{1}$ and $f_{2}$ are finished; and (2) $m>\log (q)$, meaning that $A$ managed to produce more than $\log (q)$ pairs $\left(x_{r}, y_{s}\right)$ resulting in the same value $z=f_{1}\left(x_{r}\right) \oplus f_{2}\left(y_{s}\right)$.

(Interestingly, this parameter $m$ corresponds to the largest "multi-collision" generated by $A$ in the "filling" stage. As argued by Shrimpton and Stam [17] for the case of truly random functions $f_{i}$ and $q \approx 2^{n / 2}$, the value $m$ must be smaller than $n^{1+o(1)} \approx \log q$ with high probability, more or less corresponding to saying that the attacker $A$ must almost always be in case (1).)

By assumption that $A$ succeeds to find a collision with probability $\geq \varepsilon$, at least one of these complementary cases happens with probability $\geq \varepsilon / 2$.

Case (1): A finds a collision and $m \leq \log (q)$. This is the "easy" case. Intuitively, by querying at most $q$ bins $z$ in the second stage, $A$ learned the value of $F$ in 
at most $q m \leq q \log (q)$ points $(x, y)$. As we will see, it will allow $B$ to guess the colliding points $(x, y),\left(x^{\prime}, y^{\prime}\right)$ with probability $1 /(q \log (q))^{2}$, and then forge the value $f_{3}\left(z^{\prime}\right)=f_{3}(z) \oplus f_{1}(x) \oplus f_{1}\left(x^{\prime}\right)$. More formally, $B$ starts by choosing two random indices $j<i$ between 1 and $q$. Let $z_{i}, z_{j}$ be the $i$-th and $j$-th queries made to $f_{3}$. When the query $f_{3}\left(z_{i}\right)$ is made, $B$ chooses random elements $\left(x_{i}, y_{i}\right) \in \operatorname{Bin}\left(z_{i}\right)$ and $\left(x_{j}, y_{j}\right) \in \operatorname{Bin}\left(z_{j}\right)$ (assuming these sets are nonempty, otherwise $B$ gives up), and predicts that $f_{3}\left(z_{i}\right)=f_{1}\left(x_{i}\right) \oplus f_{1}\left(x_{j}\right) \oplus f_{3}\left(z_{j}\right)$. Notice, this corresponds to guessing that $F\left(x_{i}, y_{i}\right)=F\left(x_{j}, y_{j}\right)$, which implies that $A$ is about to find a collision. This strategy cannot be successful unless $A$ finds a collision (which we are assuming happens in this case), and unless the colliding bins $z_{i}$ and $z_{j}$ are distinct, which we also argued earlier as following from our No Collision assumption. But when $A$ does find a collision, $B$ 's chance of guessing the indices $i, j$ correctly is $1 /\left(\begin{array}{c}q \\ 2\end{array}\right) \geq 1 / q^{2}$. Moreover if $\max _{z}|\operatorname{Bin}(z)| \leq \log (q)$, $B$ 's chance of guessing the right elements $\left(x_{i}, y_{i}\right)$ and $\left(x_{j}, y_{j}\right)$ in $\operatorname{Bin}\left(z_{i}\right)$ and $\operatorname{Bin}\left(z_{j}\right)$ is at least $1 / \log (q)$ each. Thus $B$ 's chance of success with this strategy is at least $1 / q^{2} \log ^{2}(q)$ when $\max _{z}|\operatorname{Bin}(z)| \leq \log (q)$ and $A$ finds a collision.

Case (2): A produces $m>\log (q)$. This is the "hard" case, where our ballsand-bins terminology comes in handy. Intuitively, if $A$ throws $q^{2}$ balls with a guarantee that some bin will contain a lot of balls at the end, $B$ should have a non-trivial chance (analyzed below) to guess the bin $z$ corresponding to some ball $(x, y)$ before this ball is thrown. To effect such a guess, when $A$ "throws" the ball $(x, y)$ by querying, say, $f_{1}(x)$ after previously querying $f_{2}(y), B$ can predict that $f_{1}(x)=z \oplus f_{2}(y)$, or conversely with $f_{1}$ and $f_{2}$ reversed if $A$ queries $f_{2}(y)$ after querying $f_{1}(x)$. In other words, predicting the output of $f_{1}$ or $f_{2}$ on a value queried by $A$ is equivalent to predicting the bin where a particular ball $(x, y)$ will land at the point when the latest of the two queries $f_{1}(x), f_{2}(y)$ is made. Thus, we may view $B$ 's task as consisting of observing a set of $Q=q^{2}$ balls being placed by groups in $2^{n}$ bins, and interrupting the game at some point to predict the bin where a particular ball that is about to be placed. We model this by a "balls-and-bins" game played by $A$ and $B$, where $A$ is incrementally throwing $Q$ balls into bins, trying to fill some bin with more than $\log (q)$ balls, and yet without having $B$ be able to guess the position of a ball before it is placed. Based on our discussion, the precise "rules" of this game are as follows:

\section{BALLS-AND-Bins GAmE:}

- The game proceeds in $2 q$ rounds, after which $A$ is required to throw exactly $Q=q^{2}$ balls.

- Before each round, $A$ announces to $B$ at most $q$ balls $b_{1}, \ldots, b_{t}$ that it will be throwing into (necessarily) distinct bins in this round. [Intuitively, a round corresponds to a query to $f_{1}\left(x_{r}\right)$ (or $f_{2}\left(y_{s}\right)$ ), and the balls are the corresponding values $\left(x_{r}, y_{j}\right)$ (or $\left.\left(x_{i}, y_{s}\right)\right)$ for prior $x_{i}$ 's or $y_{j}$ 's.]

- In turn, $B$ can secretly "pass" or make a "guess" $(\ell, z)$ that the ball $b_{\ell}$ will be thrown into bin $z$ (where $1 \leq \ell \leq t$ ). [Intuitively, a successful guess will allow $B$ to forge either $f_{1}$ or $f_{2}$, as outlined earlier.] 
- $A$ announces to $B$ the bins where $b_{1} \ldots b_{t}$ are thrown. [Intuitively, $B$ learns the value of $f_{1}$ or $f_{2}$ at the queried point, allowing it to learn the bin identities.]

- If $B$ made a guess during this round, $B$ wins the game if the guess is correct, and loses otherwise. If $B$ did not make a guess, proceed to the next round.

- $B$ must make a guess at some round, while $A$ must fill at least one bin with more than $(\log q)$ balls.

Lemma 1. Irrespective of $A$ 's strategy, there exists an efficient strategy for $B$ to win the above game with probability at least $1 / 4 q^{2}$ whenever some bin contains more than $\log (q)$ balls at the end of the game.

Proof. B's strategy is relatively simple:

1. Choose a random index $i$ between 1 and $q^{2}$, and a second random integer $k$ between 1 and $\log (q)$.

2. Pass in all the rounds before the $i$-th overall ball is about to be thrown.

3. When the $i$-th ball is about to be thrown, make a secret guess that this ball will be thrown in a random bin $z$ chosen among those bins already containing at least $k$ balls prior to this round (or guess any bin if no such bin exists).

We argue that with this strategy, $B$ 's chance of success is at least $1 / 4 q^{2}$, provided that some bin contains more than $\log (q)$ balls by the end of the game. Let $c_{j}$ be the total number of balls that are thrown into bins that already have at least $j$ balls in them right before the round when this ball is thrown. Thus $c_{0}=q^{2}$ and $c_{\log (q)} \geq 1$ by assumption that a "heavy" bin exists at the end of the game. Also note that $c_{j}$ is an upper bound for the number of bins that have $j+1$ balls in them at the end of the game, since for a bin to receive $j+1$ balls, some ball has to be thrown into it when the bin already has $j$ balls.

For a fixed value of $k, B$ 's chance of correctly guessing the bin is at least $\frac{c_{k}}{q^{2}} \cdot \frac{1}{c_{k-1}}=\frac{1}{q^{2}} \cdot \frac{c_{k}}{c_{k-1}}$. This is because $B$ has chance at least $\frac{c_{k}}{q^{2}}$ of choosing a ball thrown into a bin with at least $k$ balls, and then has at least chance $\frac{1}{c_{k-1}}$ of choosing the bin correctly, given that there are at most $c_{k-1}$ bins with $k$ balls in them even at the end of the game, let alone in some intermediate round. Summing over the different values of $k$ (which each have chance $1 / \log (q)$ of being selected), we thus see that $B$ 's chance of success is

$$
\begin{aligned}
\sum_{k=1}^{\log (q)} \frac{1}{\log (q)} \cdot \frac{1}{q^{2}} \cdot \frac{c_{k}}{c_{k-1}} & =\frac{1}{q^{2}} \text { ArithmeticMean }\left(\frac{c_{1}}{c_{0}}, \ldots, \frac{c_{\log (q)}}{c_{\log (q)-1}}\right) \\
& \geq \frac{1}{q^{2}} \text { GeometricMean }\left(\frac{c_{1}}{c_{0}}, \ldots, \frac{c_{\log (q)}}{c_{\log (q)-1}}\right) \\
& =\frac{1}{q^{2}}\left(\frac{c_{\log (q)}}{c_{0}}\right)^{\frac{1}{\log (q)}} \geq \frac{1}{q^{2}}\left(\frac{1}{q^{2}}\right)^{\frac{1}{\log (q)}} \\
& =\frac{1}{4 q^{2}}
\end{aligned}
$$

as claimed, where we used $c_{0}=q^{2}$ and $c_{\log (q)} \geq 1$. 
The above lemma immediately gives us a forger $B$ for Case (2), which succeeds to forge either $f_{1}$ or $f_{2}$ with probability at least $1 / 4 q^{2}>1 /(q \log (q))^{2}$ for that case. As $B$ chooses randomly which strategy to use and one of the two cases must occur with probability at least $\varepsilon / 2, B$ 's chance of success is at least $\frac{\varepsilon}{2} \min \left(1 /(q \log (q))^{2}, 1 / 4 q^{2}\right)=\varepsilon / 2(q \log (q))^{2}$, completing the WCR proof under our two simplifying assumptions.

General Case. Note that our (main) Query Order Assumption (namely that queries to $f_{3}$ come before queries to $f_{1}$ and $f_{2}$ ) is only used rather weakly, in the sense that $A$ could make its queries in any order as long as the query which completes the collision is a query to $f_{3}$. Thus removing this assumption amounts to handling two extra cases, in which collisions are completed with queries to $f_{1}$ or $f_{2}$ instead of $f_{3}$. It turns out these cases can be handled fairly similarly to the $f_{3}$ case. The details are deferred to the full version [9].

\section{Security of SS-NMAC as a PRF}

In this section we show that SS-NMAC is a secure PRF if $f$ is a secure PRF. We will prove a stronger property in Section 6. here we give a proof reducing to the security of encrypted CBC-MAC, which gives a weaker result but a better security bound. The precise statement is the following theorem.

Theorem 3. Let $f:\{0,1\}^{\kappa} \times\{0,1\}^{n} \rightarrow\{0,1\}^{n}$ and let $H$ be the SS-NMAC function family. Then, letting $q=\mu / n$ and $\varepsilon=\operatorname{InSec}_{f}^{\text {prf }}(t, q, q n)$, we have

$$
\operatorname{InSec}_{H}^{\mathrm{prf}}(t, \tilde{q}, \mu) \leq 5 q^{2} / 2^{n}+4 \varepsilon .
$$

Proof. Let $H^{*}$ be the SS-NMAC construction where $f_{1}, f_{2}, f_{3}, f_{4}$ are random functions. Then obviously $\operatorname{InSec}_{H}^{\mathrm{prf}}(t, \tilde{q}, \mu) \leq \operatorname{InSec}_{H^{*}}^{\mathrm{prf}}(t, \tilde{q}, \mu)+4 \varepsilon$, so it suffices to show that $\operatorname{InSec}_{H^{*}}^{\text {prf }}(t, \tilde{q}, \mu) \leq 5 q^{2} / 2^{n}$ where $q=\mu / n$.

We show that $\operatorname{InSec}_{H^{*}}^{\text {prf }}(t, \tilde{q}, \mu) \leq 5 q^{2} / 2^{n}$ by reducing to the security of the "original" encrypted CBC-MAC, which is defined using a function family $f$ of $n$-bit to $n$-bit functions by

$$
C\left[f_{1}, f_{2}\right]\left(x_{1}\|\ldots\| x_{m}\right)=f_{2}\left(\ldots f_{1}\left(f_{1}\left(x_{1}\right) \oplus x_{2}\right) \ldots\right)
$$

Let $C^{*}$ be the instance of $C$ where $f_{1}, f_{2}$ are random functions (namely, $f$ is the set of all functions $\left.\{0,1\}^{n} \rightarrow\{0,1\}^{n}\right)$. It is known that $\operatorname{InSec}_{C^{*}}^{\text {prf }}(t, \tilde{q}, \mu) \leq q^{2} / 2^{n}$ where still $q=\mu / n$ [14]. The security proof in [14] is also easily seen to apply to the case of a three-keyed, "alternating" encrypted CBC-MAC defined by

$$
C_{A}\left[f_{2}, f_{3}, f_{4}\right]\left(x_{1}\|\ldots\| x_{m}\right)=f_{4}\left(\ldots f_{2}\left(f_{3}\left(f_{2}\left(x_{1}\right) \oplus x_{2}\right) \oplus x_{3}\right) \ldots\right)
$$

in which encryptions by $f_{2}$ and $f_{3}$ alternate. Thus $\operatorname{InSec}_{C_{A}^{*}}^{\text {prf }}(t, \tilde{q}, \mu) \leq q^{2} / 2^{n}$ where $C_{A}^{*}$ is the random function implementation of $C_{A}$.

Note that $C_{A}$ becomes $H$ if each block of input is repeated once and encrypted with a call to $f_{1}$. Thus a distinguisher $D$ for $H^{*}$ can be used to obtain a distinguisher $D^{\prime}$ for $C_{A}^{*}$ : sample a key $k_{1}$ to simulate the function $f_{k_{1}}$, then simulate 
a query $x_{1}\|\cdots\| x_{m}$ of $D$ to the oracle $H^{*}$ by passing $f_{k_{1}}\left(x_{1}\right)\left\|f_{k_{1}}\left(x_{1}\right)\right\| f_{k_{1}}\left(x_{2}\right)$ $\left\|f_{k_{1}}\left(x_{2}\right) \cdots f_{k_{1}}\left(x_{m}\right)\right\| f_{k_{1}}\left(x_{m}\right)$ to the oracle for $C_{A}^{*}$.

If the oracle is a true instance of $C_{A}^{*}$ the answers returned to $D$ look exactly as the answers of an oracle to $H^{*}$, so $D^{\prime}$ 's chance of distinguishing correctly is unaffected in that case. If on the other hand the oracle is a random function the answers returned to $D$ are independent random values except when the same input is queried twice to the random oracle, which can happen because of collisions in $f_{k_{1}}$. The chance of a collision in $f_{k_{1}}$ when $q=\mu / n$ blocks of message are queried and $f_{k_{1}}$ is a random function is at most $q^{2} / 2^{n}$, however, so $D$ and $D^{\prime}$ 's distinguishing advantages differ by at most $q^{2} / 2^{n}$. Thus, since $D^{\prime}$ uses twice the message length, we get the desired

$$
\operatorname{InSec}_{H^{*}}^{\mathrm{prf}}(t, \tilde{q}, \mu) \leq \operatorname{InSec}_{C_{A}^{*}}^{\mathrm{prf}}(t, \tilde{q}, 2 \mu)+q^{2} / 2^{n} \leq 5 q^{2} / 2^{n} .
$$

\section{Enhanced PRF Security in the Oracle Cipher Model}

In this section, we introduce (following [8]) a strictly stronger PRF security notion for block-cipher-based PRFs in the so called oracle cipher model, and show that SS-NMAC has (nearly) birthday "oracle cipher security" when instantiated with a secure PRP.

Let $H$ be a function using a fixed-key block cipher $f$ (or a small set of different fixed key block ciphers). Essentially, the oracle cipher model is designed to allow the adversary to view computation transcripts of $H$, but not including the internals of the block cipher calls. For example, one can imagine that the adversary witnesses a trusted party's computation of $H$ on various inputs, where the trusted party out-sources the block cipher calls to a smart-card, so that the secret keys remain hidden from the adversary. We argue that $H$ is a good random function if, subsequent to viewing a number of such computations, the adversary is unable to distinguish $H$ (queried on new values) from a truly random function.

Let $M^{f}$ be an oracle Turing machine implementing $H$. Before the game starts random keys are chosen for the block ciphers, a random function $h$ with same domain and range as $H$ is sampled, and a coin flipped to determine whether the adversary will be in the "real world" or "random world". We allow the adversary two types of queries: "transcript" queries and "oracle" queries. When the adversary $A$ makes a transcript query the transcript of the computation $M^{f}(x)$ is returned to $A$. When the adversary makes a oracle query (oracle queries must be distinct from transcript queries), the adversary either gets $H^{f}(x)$ or $h(x)$ depending on whether it is in the real world or the random world. The adversary wins if it can distinguish the two worlds.

We call the advantage of an adversary at winning this game the oracle $c i$ pher PRF security of $H$, denoted $\varepsilon_{\text {oprf }}$. Clearly $\varepsilon_{\text {oprf }} \geq \varepsilon_{\text {prf }}$ for the same number of queries and the same computational resources, since the adversary is free to play the oracle cipher game without making any transcript queries. Let $\operatorname{InSec}_{H}^{\text {oprf }}(t, \tilde{q}, \mu)$ be the maximum $\varepsilon_{\text {oprf }}$ over all adversaries running in time at 
most $t$, making at most $q$ queries of total (padded) length at most $\mu$, where the running time includes the time necessary to run $H$ and $M^{f}$. (Obviously, $\operatorname{InSec}_{H}^{\text {oprf }}(t, \tilde{q}, \mu)$ implicitly depends on the choice of $\left.M.\right)$ We have the following theorem showing that the oracle cipher security of SS-NMAC is nearly equivalent to its standard PRF security.

Theorem 4. Let $f:\{0,1\}^{\kappa} \times\{0,1\}^{n} \rightarrow\{0,1\}^{n}$, let $H:\{0,1\}^{4 \kappa} \times \operatorname{Dom}(H) \rightarrow$ $\{0,1\}^{n}$ be the SS-NMAC function family, and let $M^{f_{1}, f_{2}, f_{3}, f_{4}}$ be the natural oracle Turing implementation of $S S-N M A C$, which makes $3 \ell+1$ oracle calls to compute $H(x)$ on a padded input of $\ell$ blocks. Then with respect to this oracle Turing machine, and letting $q=\mu / n$ and $\varepsilon=\operatorname{InSec}_{f}^{\text {prf }}(t, q, q n)$, we have

$$
\operatorname{InSec}_{H}^{\mathrm{oprf}}(t, \tilde{q}, \mu) \leq 30 q^{2} \log ^{2}(q) / 2^{n}+4 \varepsilon \text {. }
$$

Proof. Let $H^{*}$ be the instantiation of $H$ with a truly random function family instead of with $f$. We clearly have $\operatorname{InSec}_{H}^{\text {oprf }}(t, \tilde{q}, \mu) \leq \operatorname{InSec}_{H^{*}}^{\text {oprf }}+4 \varepsilon$, so it suffices to show $\operatorname{InSec}_{H^{*}}^{\text {oprf }} \leq 30 q^{2} \log ^{2}(q) / 2^{n}$.

We now modify the game like so: for each type of query (transcript and oracle), the adversary is allowed to view the transcript of the computation of $H^{*}(x)$ up to the application of $f_{4}$. Then for a transcript query the actual application of $f_{4}$ is shown as part of the transcript to the adversary, whereas for an oracle query the value of the oracle query is simply appended to the transcript (which will be the value of $f_{4}$ in the real world, or else simply the value of the random function $h)$. Note the adversary knows in either case which type of query it is witnessing, but cannot independently verify $f_{4}$ for oracle queries unless it happens to make another query later (either transcript or oracle) which results in the same input to $f_{4}$. In fact, if the adversary never makes two queries at least one of which is an oracle query that result in the same input to $f_{4}$, the two worlds look exactly alike (because $f_{4}$ is uniformly random) and the adversary has zero advantage.

Thus the adversary's advantage is upper bounded by its probability of finding a collision at the input to $f_{4}$ with free oracle access to $f_{1}, f_{2}, f_{3}$, which is in turn upper bounded by the collision resistance of the Shrimpton-Stam compression function when instantiated with random functions. Thus Theorem 1 applied with MAC insecurity $1 / 2^{n}$ gives $\operatorname{InSec}_{H^{*}}^{\text {oprf }}(t, \tilde{q}, \mu) \leq 30 q^{2} \log ^{2}(q) / 2^{n}$, as desired.

\section{Unpredictability vs. Pseudorandomness}

Given that our solution is three times slower than CBC-MAC, it is interesting to see if existing block ciphers, such as AES, are indeed more unpredictable than pseudorandom. Notice, even if our $n$-bit block cipher is completely ideal, it has security $\varepsilon_{\text {prf }} \sim q^{2} / 2^{n+1}$ as a one-block PRF, and a much better security $\varepsilon_{\text {mac }} \sim$ $1 /\left(2^{n}-q\right)$ as a one-block MAC, where $q$ is the number of input queries issued by the attacker. Also, in theory is is trivial to construct artificial block ciphers which are much more unpredictable than pseudorandom. Unfortunately, existing block ciphers are neither ideal nor artificial. For such "real" block ciphers, to the best 
of our knowledge, this gap between unpredictability and pseudorandomness has not been researched extensively. In part, this might be due to the cryptanalytic "culture" to call the attack truly "successful" if it actually recovers the secret key, which, obviously, will not demonstrate the gap we are seeking here.

We give a (rather weak) example to demonstrate this point. It is well known in complexity theory [20] that no pseudorandom generator with $\kappa$-bit key can have security more than $2^{-\kappa / 2}$ (against non-uniform attackers), even against linear tests 3 This means that no non-trivial PRF with a $\kappa$-bit key can have security $\varepsilon_{\text {prf }} \leq 2^{-\kappa / 2}$, even for $q=O(1)$ (e.g., AES cannot be more than $2^{-64}$ secure, even for $q=2$ !). In contrast, no such limitation is known for unpredictability, even for exponentially high number of queries $q$ (e.g., for all we know, AES might be almost $2^{-128}$ secure, even for $q=2^{30}$ or higher). However, the above theoretical "separation" is not considered a "real attack", since the best known way to translate this specific $2^{-\kappa / 2}$ distinguishing attack to the key recovery attack takes time $\Omega\left(2^{\kappa}\right)$, which is trivial.

We hope that our work will motivate further research to understand the gap between unpredictability and pseudorandomness of existing block ciphers, such as AES. In particular, to answer the question if existing modes, such as CBCMAC or HMAC, should be replaced by slower, but more "resilient" modes, such as SS-NMAC.

\section{References}

1. Alon, N., Goldreich, O., Hastad, J., Peralta, R.: Simple Construction of Almost k-wise Independent Random Variables. Random Struct. Algorithms 3(3), 289-304 (1992)

2. An, J.H., Bellare, M.: Constructing VIL-MACs from FIL-MACs: Message Authentication under Weakened Assumptions. In: Wiener, M. (ed.) CRYPTO 1999. LNCS, vol. 1666, pp. 252-269. Springer, Heidelberg (1999)

3. Bellare, M.: New Proofs for NMAC and HMAC: Security without CollisionResistance. In: Dwork, C. (ed.) CRYPTO 2006. LNCS, vol. 4117, pp. 602-619. Springer, Heidelberg (2006)

4. Bellare, M., Kilian, J., Rogaway, P.: The Security of Cipher Block Chaining. In: Desmedt, Y.G. (ed.) CRYPTO 1994. LNCS, vol. 839, pp. 341-358. Springer, Heidelberg (1994)

5. Bellare, M., Canetti, R., Krawczyk, H.: Pseudorandom Functions Re-visited: The Cascade Construction and Its Concrete Security. In: FOCS 1996, pp. 514-523 (1996)

6. Bellare, M., Canetti, R., Krawczyk, H.: Keying Hash Functions for Message Authentication. In: Koblitz, N. (ed.) CRYPTO 1996. LNCS, vol. 1109, pp. 1-15. Springer, Heidelberg (1996)

7. Dodis, Y., Pietrzak, K., Puniya, P.: A New Mode of Operation for Block Ciphers and Length-Preserving MACs. In: Smart, N.P. (ed.) EUROCRYPT 2008. LNCS, vol. 4965, pp. 198-219. Springer, Heidelberg (2008)

${ }^{3}$ Since an $\varepsilon$-secure pseudorandom generator must also be an $\varepsilon$-biased set [12, and such sets must have seed length at least $2 \log (1 / \varepsilon)$ (see [1]). Thus, $\kappa<2 \log (1 / \varepsilon)$. 
8. Dodis, Y., Puniya, P.: Feistel Networks Made Public, and Applications. In: Naor, M. (ed.) EUROCRYPT 2007. LNCS, vol. 4515, pp. 534-554. Springer, Heidelberg (2007)

9. Dodis, Y., Steinberger, J.: Message Authentication Codes from Unpredictable Block Ciphers. Full version of this paper, http://people.csail.mit.edu/dodis/ps/tight-mac.ps

10. Goldreich, O., Levin, L.: A hard-core predicate for all one-way functions. In: STOC 1989, pp. 25-32 (1989)

11. Luby, M., Rackoff, C.: How to construct pseudo-random permutations from pseudorandom functions. SIAM J. Comput. 17(2), 373-386 (1988)

12. Naor, J., Naor, M.: Small-Bias Probability Spaces: Efficient Constructions and Applications. SIAM J. Comput. 22(4), 838-856 (1993)

13. Naor, M., Reingold, O.: From unpredictability to indistinguishability: A simple construction of pseudo-random functions from MACs. In: Krawczyk, H. (ed.) CRYPTO 1998. LNCS, vol. 1462, pp. 267-282. Springer, Heidelberg (1998)

14. Petrank, E., Rackoff, C.: CBC MAC for Real-Time Data Sources. J. Cryptology $13(3), 315-338$ (2000)

15. Preneel, B., van Oorschot, P.C.: MDx-MAC and Building Fast MACs from Hash Functions. In: Coppersmith, D. (ed.) CRYPTO 1995. LNCS, vol. 963, pp. 1-14. Springer, Heidelberg (1995)

16. Rogaway, P., Steinberger, J.: How to Build a Permutation-Based Hash Function. In: Wagner, D. (ed.) CRYPTO 2008. LNCS, vol. 5157, pp. 433-450. Springer, Heidelberg (2008)

17. Shrimpton, T., Stam, M.: Building a Collision-Resistant Compression Function from Non-Compressing Primitives. In: Aceto, L., Damgård, I., Goldberg, L.A., Halldórsson, M.M., Ingólfsdóttir, A., Walukiewicz, I. (eds.) ICALP 2008, Part II. LNCS, vol. 5126, pp. 643-654. Springer, Heidelberg (2008)

18. Simon, D.R.: Finding Collisions on a One-Way Street: Can Secure Hash Functions Be Based on General Assumptions? In: Nyberg, K. (ed.) EUROCRYPT 1998. LNCS, vol. 1403, pp. 334-345. Springer, Heidelberg (1998)

19. Wang, X., Yu, H.: How to break MD5 and Other Hash Functions. In: Cramer, R. (ed.) EUROCRYPT 2005. LNCS, vol. 3494, pp. 19-35. Springer, Heidelberg (2005)

20. Zuckerman, D.: Private communication 